\title{
Complicaciones del uso de la heroína parenteral: artículo de revisión
}

Fecha de recibido: 15 de enero de 2016 Fecha de aprobación: 22 de agosto de 2016
Forma de citar este artículo: Toro JH, Álvarez ML. Complicaciones del uso de la heroína parenteral: artículo de revisión. Med U.P.B. 2017;36(2):146-152. DOI:10.18566/medupb.v36n2.a07

1. Enfermero, epidemiólogo. Docente, Facultad de Enfermería, Universidad de Antioquia. Medellín, Colombia.

2. Enfermera, especialista en farmacodependencia. Docente investigadora, Escuela de Ciencias de la Salud, Universidad Pontificia Bolivariana. Medellín, Colombia.

Dirección de correspondencia: Matilde Ligia Álvarez Gómez. Correo electrónico: matilde. alvarez@upb.edu.co

\section{Parenteral heroin complications: Article review / Complicações do uso da heroína parenteral: artigo de revisão}

\author{
Jaime Horacio Toro Ocampoํㅗㄹ Matilde Ligia Álvarez Gómez²
}

\section{RESUMEN}

Revisión narrativa que buscó recopilar informacion sobre complicaciones del uso de la heroína parenteral. Fueron captados artículos científicos, revisiones y casos clínicos en las bases de datos Scielo, Lilacs, Medline. La heroína es una sustancia psicoactiva y su uso tiene consecuencias adversas para la salud física y mental; las complicaciones físicas más frecuentes son infecciones por virus como el HIV, las hepatitis B y C, infecciones de piel, tuberculosis, embolias pulmonares y enfermedades renales. Otras complicaciones frecuentes son los trastornos mentales como depresión, trastornos de personalidad y el uso concomitante de otras sustancias, como el cannabis y la cocaína. Para el profesional de enfermería es importante conocer las complicaciones del uso de la heroína para planear procesos integrales y elaborar programas de reduccion de riesgos y daños derivados del uso de la sustancia.

Palabras clave: heroína; abuso de sustancias por vía intravenosa; impactos en la salud.

\section{ABSTRACT}

This literature review sought to collect information on complications of parenteral use of heroin. Scientific articles, reviews, and case reports were selected from Scielo, Lilacs, and Medline databases. Heroin is a psychoactive substance and its use has mental and physical health consequences; among the most common physical consequences are blood-borne infectious diseases such as HIV, hepatitis B and C, skin infections, tuberculosis, pulmonary embolism, and renal disease. Other frequent complications include mental disorders, such as depression, personality disorders, and the concomitant abuse of other substances, such as cannabis and cocaine. It is important for nurses to be aware of the complications of heroin use in order to plan comprehensive processes and develop nursing programs that reduce the risks and harm resulting from use of the substance.

Keywords: heroin; substance abuse, intravenous; impacts on health.

\section{RESUMO}

Revisão narrativa que buscou recopilar informação sobre complicações do uso da heroína parenteral. Foram captados artigos científicos, revisões e casos clínicos nas bases de dados Scielo, Lilacs, Medline. A heroína é uma substância psicoativa e seu uso tem consequências adversas para a saúde física e mental; as complicações físicas mais frequentes são infecções por vírus como o HIV, as hepatite B e C, infecções da pele, tuberculose, embolias pulmonares e doenças renais. Outras complicações frequentes são os transtornos mentais como depressão, transtornos de personalidade e o uso concomitante de outras substâncias, como a maconha e a cocaína. Para o profissional de enfermagem é importante conhecer as complicações do uso da heroína para planejar processos integrais e elaborar programas de redução de riscos e estragos derivados do uso da substância.

Palavras chave: heroína; abuso de substâncias por via intravenosa; impactos na saúde. 


\section{INTRODUCCIÓN}

El uso del opio data de la antigüedad, ya en Alejandría, en el antiguo Egipto, era usado con fines recreativos, religiosos y medicinales ${ }^{1}$. La heroína fue sintetizada en 1886 a partir de la acetilación de la morfina; este fármaco fue comercializado como un sedante, antitusígeno y como sustituto de la morfina con la creencia errónea de que era menos adictiva. En la década de los 70 el uso de heroína en Europa empezó a constituirse en un problema de salud pública, ya que se inició la administración por vía parenteral, lo que condujo a epidemia de Hepatitis B, entre otras. En España, en el periodo comprendido entre 1988 y 1989 el 41\% de los heroinómanos que usaban la droga venosa tenía HIV (Virus de la Inmunodeficiencia Humana) positivo ${ }^{2}$. En este país, en personas que asitían a terapia de remplazo con metadona, la prevalencia de esta infeccion fue de $43.5 \%$. Se calcula que en el mundo los consumidores de opiáceos están entre 15 y 21 millones, la mitad de ellos están en Asia ${ }^{3}$. En Medellin (Colombia) la prevalencia de HIV en habitantes de calle es de $7.8 \%$; de estos $12 \%$ está conformado por hombres y $2.9 \%$ por mujeres ${ }^{4}$.

En poblacion general colombiana 31852 personas han consumido heroína alguna vez en la vida. De ellas, 7011 consumieron esta sustancia en el último año y 3 592 lo hicieron en el último mes. Cabe destacar que la problemática puede ser superior en número de personas por el uso clandestino que es difícil de calcular ${ }^{5}$.

Las consecuencias médicas del uso crónico de heroína inyectada incluyen venas cicatrizadas o colapsadas, flebitis, abscesos y otras infecciones de los tejidos blandos, enfermedades hepáticas y renales. Las complicaciones pulmonares pueden ser el resultado de la mala salud del adicto, así como de los efectos depresores de la heroína sobre el sistema respiratorio. Las reacciones inmunes a los contaminantes pueden causar artritis u otros problemas reumatológicos ${ }^{6,7}$.

Las personas adictas a heroína con frecuencia pueden sufrir de otros trastornos mentales, entre ellos están: los trastornos de personalidad y específicamente el antisocial 25\%-40\% y la depresión 50\%-75\% ${ }^{7-10}$. Algunos clínicos afirman que el deterioro social precoz que presentan los adictos a la heroína se debe, principalmente, a la comorbilidad psiquiátrica y de manera secundaria a la conducta de dependencia ${ }^{10}$.

Los consumidores tienen gran deterioro social, la mayoría carece de vivienda, mendigan o incluso roban, están criminalizados, estigmatizados y discriminados. Esos factores, combinados con una pobre autoestima y una escasa confianza en las autoridades, hacen de ellos una población a la que los servicios sociales y sanitarios tradicionales tienen poco acceso y, por ende, se agravan los problemas mencionados ${ }^{11}$.
El uso de heroína inyectable trae consecuencias adversas para la salud pública, debido a la propagación de infecciones y de trastornos mentales que induce. $\mathrm{La}$ marginalidad social que sufren estas personas conduce al microtráfico, a la delincuencia y a la prostitución, lo que produce costos para los sistemas de salud. E1 profesional de enfermería, por su formación en las disciplinas biológica y social, interviene en el cuidado de los heroinómanos en todas las fases del tratamiento que incluye rehabilitación y disminución de riesgos y daños. Este artículo es importante para la profesión porque incrementará los conocimientos sobre las complicaciones ocasionadas por esta droga para brindar un cuidado de enfermería de alta calidad.

\section{Dependencia a opiáceos}

Los opiáceos son un grupo de fármacos con gran actividad analgésica, derivados naturales y sintéticos del opio, con características farmacológicas comunes como son: la actividad analgésica, sedación y sueño. Inducen depresión respiratoria y tienen potencial de tolerancia y dependencia ${ }^{12}$.

Todo individuo está dotado de un sistema de opioides endógenos que derivan de tres polipéptidos precursores, la proencefalina de la que deriva las encefalinas, la prodinorfina de la que se sintetizan las dinorfinas y las neoendorfinas y, por último, la propiomelanocortina de la que derivan las betaendorfinas. Tienen, además, subtipos de receptores acoplados a proteínas $G(\mu 1,2$, kappa 1 , 2,3 , delta 1,2 , sigma y épsilon) con amplia distribución en el Sistema Nervioso Central (SNC) y periférico. Los opiáceos se clasifican dentro del grupo de depresores del $\mathrm{SNC}$ y producen dependencia física y psíquica. Generan refuerzo positivo al activar vías dopaminérgicas mesolímbicas y mesocorticales, especialmente en área ventral tegmental (AVT) y el núcleo accubens (NAc). Aunque la activación de los sistemas dopaminérgicos parece tener la misma base que en el caso de la cocaína, los mecanismos son diferentes para los opiáceos. Los opiáceos activan este sistema e inhiben las neuronas que tienen acción inhibitoria sobre neuronas dopaminérgicas en el AVT, o activando los receptores opioides presinápticos, lo que facilita la liberación de dopamina. Por su parte la cocaína incrementa la actividad dopaminérgica por inhibición de la recaptación de dopamina ${ }^{12,13}$.

La heroína (diacetilmorfina) es el opiáceo que actúa rápido en SNC. Llega al cerebro en 15-30 segundos lo que aumenta el riesgo de dependencia. Adicionalmente, produce una fuerte inhibición de la actividad eléctrica neuronal y liberación de neurotransmisores, así como recompensa y euforia por acción dopaminérgica, que es su principal propiedad reforzadora ${ }^{12,13}$. 
La heroína provoca a los pocos segundos una sensación de subida o "flash" que consiste en una sensación subjetiva muy agradable de calor con goce intenso, posteriormente euforia y relajación, que tiene una duración de 5-15 minutos, seguida del llamado flooding o profunda sensación de bienestar y plenitud con somnolencia, letargia y enlentecimiento motor que dura de cuatro a ocho horas ${ }^{13,14}$.

La diacetilmorfina se incluye en el grupo de depresores del SNC. La intoxicación por esta sustancia puede ser mortal por la depresión respiratoria. La triada de la intoxicación se caracteriza por miosis, disminución del patrón respiratorio y deterioro del estado de conciencia. El síndrome de abstinencia aparece al suspender o reducir la dosos de la droga, se presenta por la disminución de los niveles de proencefalinas y metencefalinas, con un aumento de las dinorfinas que estimulan los receptores $\mathrm{k}$ e inhiben las neuronas dopaminérgicas, lo que desencadena manifestaciones físicas y motivacionales. El síndrome de abstinencia tiene tres etapas: en la primera aparece rinorrea, lagrimeo, bostezos, ansiedad y sudoración; en la segunda se presenta midriasis, piloerección, dolores y contracturas musculares, diaforesis, ansiedad e irritabilidad; finalmente, en la fase tardía, se presentan espasmos musculares e intestinales, vómito y diarrea ${ }^{12,15}$.

\section{Complicaciones infecciosas del consumo de heroína}

Según el programa conjunto de las Naciones Unidas sobre el VIH/SIDA (Onusida) el número de personas con el HIV se estima en 37.3 millones $^{16}$. Las personas que usan drogas parenterales son el grupo más afectado por HIV: la prevalencia de esta infección en drogadictos es 22 veces superior que en la población general ${ }^{17}$.

La inyección de heroína es el método que tiene más riesgo para la salud puesto que se transmiten infecciones por el uso compartido de agujas y jeringas contaminadas, lo que aumenta la probabilidad de que los adictos se infecten con virus como el VIH, el de la hepatitis B o el de la hepatitis C. En personas que usan drogas inyectables se ha reportado prevalencia de SIDA de $24 \%$ a $28 \%{ }^{18-19}$. El uso de sustancias psicoactivas (SPA) parenterales es de mayor riesgo para el SIDA que tener relaciones sexules con homosexuales ${ }^{20}$. Las mujeres son más suceptibles a sufrir HIV, sobre todo en poblaciones marginadas y en países pobres. En Kenia se halló que las mujeres que se inyectaban SPA tenían más de cuatro veces la probabilidad de ser VIH positivo en comparación con los hombres ${ }^{21}$. Un estudio realizado en barrios pobres de Nueva York encontró asociación entre ser mujer y sufrir HIV $(\mathrm{RRA}=2.14)^{22}$.

Los que se inyectan la heroína tienen el doble de riesgo de contraer HIV que los que la inhalan. Algunos adictos cambian el uso de la heroína inyectada por otras forma de uso como inhalada y los argumentos para este cambio son: mejorar la autoimagen, disminuir la estigamtizacion social y mejorar la salud ${ }^{23}$.

Se han descrito casos de otras infecciones, por ejemplo: botulismo en un hombre de 29 años de edad, adicto a heroína que tenía múltiples heridas de piel infectadas por el uso parenteral de la droga ${ }^{24}$. También es frecuente hallar fibrosis venosa, trastornos de la temperatura corporal (hipotermia), sepsis, endocarditis o embolias. Muchas de estas complicaciones se deben a la contaminación por otras sustancias con las que se mezcla la heroína, por ejemplo: la quinina, la maltosa, el almidón, la harina; estas sustancias producen una reacción de cuerpo extraño por su insolubilidad y pueden dañar los órganos ${ }^{10,25,26}$.

El uso de jeringas contaminadas y la combinación con otras sustancias son responsables de múltiples infecciones, por ejemplo por Estafilococus Aureus ${ }^{27}$ que afecta la piel, los tejidos blandos o los pulmones y es una de las causas más comunes de hospitalización y de muerte en estas personas ${ }^{28,29}$. El consumo de heroína venosa está relacionada con edema pulmonar, neumonitis por aspiración, neumonía, absceso pulmonar o embolia pulmonar séptica ${ }^{30}$.

Otra complicación pulmonar es la coinfección con tuberculosis (TB). La prevalencia de esta infección en personas con antecedentes de consumo de heroína parenteral que llevan consumiendo de ocho a 11 años es del 40\%. En los que llevan más de 11 años la prevalencia es de $66.7 \%{ }^{31}$. La prevalencia ponderada de $\mathrm{TB}$ en consumidores de heroína en Costa de Marfil fue de $1.8 \%$ y estuvo asociada a vivir en la calle en el 6.2\% (p $=0.03$ ). En cárceles españolas, la tuberculosis latente está asociada con el consumo de heroína o cocaína (60\% vs $39.3 \%$ en no consumidores; $\mathrm{p}=0.02)^{32}$.

Se han descrito casos con otras complicaciones infecciosas por el uso de heroína, como son la endocarditis tricuspídea y embolismo pulmonar séptico, que se inicia con dificultad respiratoria ${ }^{33}$. Otro reporte hace alusión a granulocitosis pulmonar después de la aplicación venosa de heroína contaminada con sustancias desconocidas ${ }^{34}$. También se ha reportado miocarditis con infiltrados leucocitarios insterticial en 21 personas adictas a opiáceos parenterales que fallecieron. Estas células eran cinco veces más abundantes en los cadáveres de los adictos que en los fallecidos por otras causas como el trauma ${ }^{35}$.

\section{Complicaciones no infecciosas del uso de heroína}

El consumo crónico de heroína produce deterioro físico. El 78.5\% de los heroinómanos de Medellín, reporta pérdida de peso, cansancio, insomnio, disminución de la 
temperatura corporal ${ }^{36}$. Muchas de las complicaciones no infecciosas del uso de heroína parenteral se deben a la toxicidad de la sustancias, a la vía de aplicación y a la mezcla con otras sustancias ${ }^{37}$. Se ha descrito la relación entre el asma bronquial y el consumo de heroína por vía inhalatoria calentada sobre papel de aluminio (Chasing the Dragon). El episodio asmático puede ser grave y requerir ventilación mecánica e, incluso, provocar la muerte. Las sustancias que adulteran la heroína y los vapores de aluminio que se desprenden al recalentarla provocan hiperreactividad bronquial ${ }^{38}$.

La adicción a opiáceos contribuye a la progresión del daño renal y provoca nefropatías. Los efectos son crónicos e irreversibles pero, a veces, agudos con una posible recuperación. Las complicaciones renales por abuso de drogas son cada vez más frecuentes y pueden abarcar un amplio espectro glomerular.El daño renal y el abuso de heroína se asocia con síndrome nefrótico, glomerulonefritis aguda, amiloidosis, nefritis intersticial y rabdomiolisis; el daño implica cambios hemodinámicos renales, en la síntesis y la degradación de la matriz glomerular y la inducción de la aterogénesis renal. La insuficiencia renal aguda se desarrolla en el 30\% de los casos de rabdomiolisis. El consumo de la sustancia está implicado por la fisiopatología de la enfermedad ${ }^{39,40}$.

Algunas complicaciones del uso de heroína son las neurológicas y se encuentran solas o comorbidas con otras enfermedades, como es el caso de un joven que fumó por primera vez heroína y sufrió de una mielitis tranversa y una rabdomiosis. Los síntomas iniciales consistieron en incapacidad para mover las piernas y se evidenció paraplejia, disminución de la fuerza en ambos brazos y anuria. Los exámenes de sangre reportaron urea en sangre de $145 \mathrm{mg} / \mathrm{dl}$, cretinina de $6 \mathrm{mg} / \mathrm{dl}$, potasio de $5 \mathrm{mEq} / \mathrm{dl}^{41}$.

Otros autores reportan el caso de un hombre de 44 años llevado a urgencias, al ingreso tenía: depresión respiratoria, glasgow de $9 / 15$, miosis, prueba de opioides en orina positiva. Los exámenes de laboratorio mostraron que tenía insuficiencia renal aguda y rabdomiolisis. Después de 12 horas mejoró su estado de conciencia, pero se quejaba de dolor en el muslo izquierdo. Una tomografía reveló edema de la fascia del vasto izquierdo, se diagnosticó síndrome compartimental de glúteo izquierdo ${ }^{42}$.

Un reporte describió tres hombres que tuvieron leucoencefalopatía después de aspirar heroína. Presentaron los siguientes síntomas: deterioro del estado de conciencia, agresividad, rigidez y nigtamus ${ }^{43}$ Algunos reportes hacen alusión a infartos cerebrales relacionados con el consumo de heroína ${ }^{44}$.

En relación con los trastornos mentales de personas dependientes a la heroína se sabe que existen prevalencias desde $80 \%$ hasta $86.9 \% \%^{45,46}$. Los más prevalentes son los trastornos de personalidad que van de $32.9 \%{ }^{7}$ a $45 \%{ }^{47}$. Entre estos, los más comunes son el trastorno de personalidad antisocial con un 30\% y el trastorno de personalidad limítrofe en el $15.3 \%{ }^{48}$. Otras enfermedades mentales frecuentes son la depresión (prevalencias de $18 \%$ a $32 \%)$, los trastornos de ansiedad (15.3\%) y los trastornos psicóticos $(2.6 \%)^{49}$.

En Medellín se halló que un poco más de la mitad de las personas adictas a heroína habían tenido ideas suicidas y algunos habían tenido intentos serios de suicidio. Las ideas que manifestaron estuvieron vinculadas con el consumo, sobre todo con las depresiones post consumo $^{36}$ Otro estudio, en la misma ciudad, en personas que estaban en rehabilitación, reportó que el trastorno mental más frecuente fue la depresión (32.9\%), le seguían los trastornos de personalidad antisocial (15.1\%), límite (8.2\%), paranoide $(2.7 \%)$ y mixto $(1.4 \%)^{7}$

Con frecuencia se reporta dependencia a la heroína y trastornos del estado de ánimo. Los dependientes a esta sustancia tienen predisposición a presentar trastornos depresivos e ideación suicida ${ }^{50}$, aunque son más frecuentes en mujeres. Un estudio en el que se siguió un grupo de heroinómanos durante 30 meses, encontró que las mujeres eran más propensas a tener depresión mayor tanto en la línea base ( $31.1 \%$ en mujeres vs $19.8 \%$ en hombres) como cuando se evaluaron, a los 36 meses después del tratamiento $(11.9 \% \text { vs } 6.1 \%)^{51}$. Una investigación en la que se aplicó una escala dimensional se reportó mayor sintomatología depresiva en mujeres $76.7 \%$ que en hombres $75 \%$. En los hombres la depresión se asoció con el uso de mezcla de cocaína y heroína y en mujeres con el uso de cigarrillo ${ }^{52}$. En Medellín, en un estudio sobre calidad de vida de heroinómanos en un programa de mantenimiento de metadona se encontró que el 62.5\% tenía sintomatología depresiva moderada y el 25\% tenía sintomatología depresiva grave ${ }^{53}$.

En adultos se han encontrado prevalencias de consumo simultáneo de heroína y cocaína (speed-ball) en el 35.8\% de los casos, de heroína y alcohol en el 7.4\%, de heroína y cannabis en el 3.7\% y de heroína y sedantes en el 11.6\% $\%^{54}$. En un centro de atención de adictos en Medellín las sustancias más consumidas junto con la heroína fueron la marihuana y la cocaína (100\% cada una), seguidas por el alcohol (92\%) e inhalantes-pegantes $(87.5 \%)^{53}$.

\section{CONCLUSIONES}

El consumo de heroína parenteral produce problemas orgánicos, los más frecuentemente son las infecciones, que son causadas por el uso de jeringas y otros elementos utilizados para diluir la sustancia. También pueden existir otros trastornos mentales entre los que destacan la depresión y los trastornos de personalidad, que son 
independientes de la vía de uso. La vía parenteral termina siendo la más usada, tiene gran potencial adictivo y, con frecuencia, se relaciona con enfermedades mentales.

En Colombia se ha incrementado el uso de la heroína y existen programas de mantenimiento con metadona que son dirigidos por personal de enfermería y que buscan reducir el riesgo y el daño por la adicción. Estos profesionales deben conocer las complicaciones psiquiatrícas y médicas generales que ocasiona la sustancia para actuar mejor en los programas de reducción de daños y riesgo. Es importante resaltar que a los centros hospitarios están llegando enfermos heroinómanos cuyas complicaciones y cuidados de enfemería se desconcen, y que muchas veces los profesionales de esta área no han tenido formación previa. Este artículo aporta algún conocimento general sobre la problemática que ayudará a formular cuidados de enfemería integrales y de calidad.

\section{DECLARACIÓN DE CONFLICTO DE INTERESES}

Los autores declaran no tener ningun conflicto de intereses.

\section{REFERENCIAS}

1. Guerra E. Evidencias del consumo de drogas en Europa durante la prehistoria. Trastor Adict 2006; 8(1):53-61.

2. Del Río M. Programa de reducción de daños. En: Sociedad Española de Toxicología. Madrid: Panamerica; 2006. p. 485-489.

3. Sanvisens A, Rivas I, Faure E, Muñoz T, Rubio M, Fuster D, et al. Características de los pacientes adictos a la heroína admitidos en un programa de tratamiento con metadona. Med Clin (Barc). 2014; 142(2):53-58.

4. Berbesi DY, Agudelo A, Segura A, Liliana P. VIH en habitantes de calle de Medellín. Rev Fac Nac Salud Pública 2012; 30:310-315.

5. República de Colombia. Ministerio de Salud y Protección Social. Estudio nacional de consumo de sustancias psicoactivas. Santa Fé de Bogotá; 2013. p. 98-92.

6. O'Connor G, McMahon G. Complications of heroin abuse. Eur J Emerg Med 2008; 15(2):104-146.

7. Álvarez M, Suárez A, Giraldo. Características de las personas adictas a heroína en una empresa social del Estado de enero 2003 a junio de 2008. SMAD 2009; 5(1):1-17.

8. Schafer I, Eiroa-Orosa FJ, Verthein U, Dilg C, Haasen C, Reimer J. Effects of psychiatric comorbidity on treatment outcome in patients undergoing diamorphine or methadone maintenance treatment. Psychopathology 2010; 43(2):88-95.

9. Chiang SC, Chan HY, Chang YY, Sun HJ, Chen WJ, Chen CK. Psychiatric comorbidity and gender difference among treatment-seeking heroin abusers in Taiwan. Psychiatry Clin Neurosci 2007; 61(1):105-111.

10. Martin M. Complicaciones psiquiátricas en la dependencia a opiáceos. En: Lorenzo P, Ladero JM, Leza JC, Lizasoain I. Drogodependencias: farmacología. Patología. Psicología. Legislación. Madrid: Médica Panamericana; 2009.p. 183-188.

11. Observatorio español sobre drogas. Situación y tendencias de los problemas de drogas en España. Observatorio español sobre drogas (OED). Delegación del Gobierno para el Plan Nacional sobre Drogas. 2009.p. 1-231.

12. Yaksh R, Wallace M. Opioides, analgesia y tratamiento. En: Goodman LS, Brunton LL, Chabner BA, Jknollman BC. Bases farmacológicas de la terapéutica.12 ed. México: McGraw Hill; 2011.p. 481-487.

13. López R. Bases bioquímicas y moleculares de la adicción a opiáceos. En: Pérez J, Valderrama J, Cervera G, Rubio G. Tratado SET de trastornos adictivos. Madrid : Panamericana; 2006. p 222-241.

14. Álvarez M, Gómez L, Velásquez J, Ramírez C, Tirado A, Vargas A. Opiáceos. En: Álvarez M, Gómez L, Velásquez J, Ramírez C, Tirado A, Vargas A. Diagnóstico, tratamiento e intervención de las personas consumidoras de drogas. Medellín: Universidad Pontificia Bolivariana; 2011. p.24-34.

15. American Psychiatric Association. Trastornos relacionados con sustancias y trastornos adictivos. En: Guía de consulta de los criterios diagnósticos del DSM-5. Washington: Masson; 2014.p.253318.

16. Onusida. El Sida en Cifras. 2013; http://www.unaids.org/sites/default/files/media_asset/ JC2571_AIDS_by_the_numbers_es_1.pdf

17. Onusida. Onusida. Hoja Inf Mund 2016. 2013;1-3. http://www.unaids.org/es/resources/factsheet

18. Ramasamy P, Lintzeris N, Sutton Y, Taylor H, Day CA, Haber PS. The outcome of a rapid hepatitis B vaccination programme in a methadone treatment clinic. Addiction. 2010; 105(2):329-334.

19. Meade CS, McDonald LJ, Weiss RD. HIV risk behavior in opioid dependent adults seeking detoxification treatment: An exploratory comparison of heroin and oxycodone users. Am J Addict 2009; 18(4):289-293. 
20. Alfonso R, Hurtado I, Espacio A, Santos G, Tomas S. [Risk behaviours and seroprevalence to HIV, $\mathrm{HBV}$ and HCV in patients of the AIDS information and prevention center in Valencia, Spain]. Gac Sanit 1999; 13(1):16-21.

21. Syvertsen JL, Agot K, Ohaga S, Strathdee SA, Camlin CS, Omanga E, et al. Evidence of injection drug use in Kisumu, Kenya: Implications for HIV prevention. Drug Alcohol Depend 2015 151:262-266.

22. Wiewe EW, Bocour A, Kersanske LS, Bodach SD, Xia Q BS. The association between neighborhood poverty and HIV diagnoses among males and females in New York City, 2010-2011. Public Heal Rep 2016; 131(2):290-302.

23. Des Jarlais DC, Arasteh K, Perlis T, Hagan H, Heckathorn DD, Mcknight C, et al. The transition from injection to non-injection drug use: Long-term outcomes among heroin and cocaine users in New York City. Addiction 2007; 102(5):778-785.

24. Holmaas G, Gilhus NE, Gjerde IO, Lund-Tonnessen S, Langorgen J. [Wound botulism in heroin addiction]. Tidsskr Nor Laegeforen 1998; 118(28):4357-4359.

25. Adams D, Logerstedt D, Hunter-Giordano A, Axe JM, Snyder-Mackler L. Current concepts for anterior cruciate ligament reconstruction: a criterion-based rehabilitation progression. J Orthop Sport Phys Ther 2013; 42(7):601-614.

26. Berbesi D, Segura-Cardona A, Montoya-Vélez L, Mateu-Gelabert P. Consumo de heroína inyectada en Colombia y comportamientos de riesgo. Artículo Orig Salud Ment 2013; 3636(1):27-31.

27. Gainza-I Nogué S, Martínez C, Hoffman S, Burillo G, Duenas A. Intoxicación por drogas. Anales Sis San Navarra 2003; 26(supl.1):99-128.

28. Cooper HLF, Brady JE, Ciccarone D, Tempalski B, Gostnell K, Friedman SR. Nationwide increase in the number of hospitalizations for illicit injection drug use-related infective endocarditis. Clin Infect Dis 2007; 45(9):1200-1203.

29. Suaya J, Mera RM, Cassidy A, O'Hara P, Amrine-Madsen H, Burstin S, et al. Incidence and cost of hospitalizations associated with Staphylococcus aureus skin and soft tissue infections in the United States from 2001 through 2009. BMC Infect Dis 2014; 14(1):296.

30. Bishay A, Amchentsev A, Saleh A, Patel N, Travis W, Raoof S. A hitherto unreported pulmonary complication in an IV heroin user. Chest 2008; 133(2):549-551.

31. Álvarez M, Godoy P. Prevalencia de la infección tuberculosa y por el VIH en los usuarios de un programa de reducción de riesgos para usuarios de drogas por vía parenteral (UDVP). Rev Esp Salud Pública 1999; 73(3):375-381.

32. Bouscaillou J, Evanno J, Prouté $M$, Inwolé $A$, Kabran $M, N$ 'Guessan $T$, et al. Prevalence and factors associated with HIV and tuberculosis in people who use drugs in Abidjan, Ivory Coast. Int $J$ Drug Policy 2016; 30:116-123.

33. Sheu C-C, Hwang J-J, Tsai J-R, Wang T-H, Chong I-W, Huang M-S. Spontaneous pneumothorax as a complication of septic pulmonary embolism in an intravenous drug user: A case report. Kaohsiung J Med Sci 2006; 22(2):89-93.

34. Kommuri A, Madan N, Nikhil M. Pulmonary talc granulomatosis-unusual causeof pulmonary infiltrates in HIV patient. Chest 2012; 142(4).

35. Dettmeyer R, Friedrich K, Schmidt P, Madea B. Heroin-associated myocardial damagesconventional and immunohistochemical investigations. Forensic Sci Int 2009; 187(1-3):42-46.

36. Pérez GA, Vallejo GC. Comorbilidad asociada en un grupo de consumidores de heroína de Medellín. Rev Colomb Psiquiatr 2010; 39:93S-111.

37. Castaño G. Complicaciones médicas no infecciosas del uso de sustancias psicoactivas. En: Vásquez E, Olaya, A, Castaño G, Castro S. Adicciones, aspectos clínicos y psicosociales, tratamiento y prevención. CIB; Medellín. p. 97-111.

38. Izquierdo M, Martínez-Moratalla J. Broncoespasmo en relación con heroína inhalada. An Med Interna 2001; 92(2).

39. Do Sameiro Faria M, Sampaio S, Faria V, Carvalho E. Nephropathy associated with heroin abuse in Caucasian patients. Nephrol Dial Transplant 2003; 18(11):2308-2313.

40. Barparda K. Enfermedad renal crónica asociada a diacetilmorfina (heroína). Rev Med UPB 2008; 21(4):406-415.

41. Gupta A, Khaira A, Lata S, Agarwal SK, Tiwari SC. Rhabdomyolysis, acute kidney injury and transverse myelitis due to naive heroin exposure. Saudi J Kidney Dis Transpl 2011; 22(6):12231225.

42. Adrish M, Duncalf R, Diaz-Fuentes G, Venkatram S. Opioid overdose with gluteal compartment syndrome and acute peripheral neuropathy. Am J Case Rep 2014; 15:22-26.

43. Blasel S, Hattingen E, Adelmann M, Nichtwei M, Zanella F, Weidauer S. Toxic leukoencephalopathy after heroin abuse without heroin vapor inhalation: MR imaging and clinical features in three patients. Clin Neuroradiol 2010; 20(1):48-53.

44. Bolz, J, Meves S. Multiple cerebral infarctions in a young patient with heroin-induced hypereosinophilic syndrome. J Neurol Sci 2015; 356(1-2):193-195.

45. Bellver R. Patología dual: características y tratamiento. In: Patología dual: características y tratamiento. 2015. 
46. Astals M, Domingo-Salvany A, Buenaventura CC, Tato J, Vazquez JM, Martín-Santos R, et al. Impact of substance dependence and dual diagnosis on the quality of life of heroin users seeking treatment. Subst Use Misuse 2008; 43(5):612-632.

47. Darke S, Ross J, Williamson A, Teesson M. The impact of borderline personality disorder on 12-month outcomes for the treatment of heroin dependence. Addiction 2005; 100(8):1121-1130.

48. Zhong B, Xiang Y, Cao X, Li Y, Zhu J, Chiu HFK. Prevalence of antisocial personality disorder among Chinese individuals receiving treatment for heroin dependence: a meta-analysis. Shanghai Arch psychiatry 2014; 26(5):259-271.

49. Puigdollers E, Domingo-Salvany A, Brugal MT, Torrens M, Alvarós J, Castillo C, et al. Characteristics of heroin addicts entering methadone maintenance treatment: Quality of life and gender. Subst Use Misuse 2004; 39(9):1353-1368.

50. Darke S, Mills K, Teesson M, Ross J, Williamson A, Havard A. Patterns of major depression and drug-related problems amongst heroin users across 36 months. Psychiatry Res 2009; 166(1):7-14.

51. Sordo L, Chahua M, Bravo MJ, Barrio G, Brugal MT, Domingo-Salvany A, et al. Depression among regular heroin users: The influence of gender. Addict Behav 2012; 37(1):148-152.

52. Risser J, Cates A, Rehman H, Risser W. Gender differences in social support and depression among injection drug users in Houston, Texas. Am J Drug Alcohol Abuse 2010; 36(1):18-24.

53. Tirado A F, Álvarez M, Velásquez JD, Lugo L, Girado LF. Calidad de vida de pacientes con dependencia a heroína de un centro de atención de drogodependencias de Medellín. Invest Educ Enferm 2012; 30(1):33-43.

54. Ashford LS. Cómo se ven afectadas las poblaciones por el VIH y el sida. Bridge. 2006;1-4. 